\title{
Gel Warping Algorithm
}

National Cancer Institute

\section{Source}

National Cancer Institute. Gel Warping Algorithm. NCI Thesaurus. Code C70591.

A computer-implemented image processing method and apparatus for normalizing gel electrophoresis images. The method includes the steps of assigning tiepoints in a reference image and in one or more object images. The tiepoints in the object image are evaluated by comparison to a corresponding tiepoint in the reference image, and the location of the tiepoint in the object image is adjusted by slight movement to a location with respect to recognizable features in both the reference and object image. Warping functions are generated and then globally optimized. 